\title{
Ultrasonic Measurement of Temperature Rise in Breast Cyst and in Neighbouring Tissues as a Method of Tissue Differentiation
}

\author{
Barbara $\operatorname{GAMBIN}^{(1)}$, Michał BYRA ${ }^{(1)}$, Eleonora KRUGLENKO ${ }^{(1)}$, \\ Olga DOUBROVINA ${ }^{(2)}$, Andrzej NOWICKI ${ }^{(1)}$ \\ (1) Institute of Fundamental Technological Research, Polish Academy of Sciences \\ Pawińskiego 5B, 02-106 Warsaw, Poland; e-mail: bgambin@ippt.pan.pl \\ (2) Belarussian State University \\ Kurchatova 5, 220045 Minsk, Belarus; e-mail: dubrovinaov@tut.by
}

(received March 2, 2016; accepted May 5, 2016)

\begin{abstract}
Texture of ultrasound images contain information about the properties of examined tissues. The analysis of statistical properties of backscattered ultrasonic echoes has been recently successfully applied to differentiate healthy breast tissue from the benign and malignant lesions. We propose a novel procedure of tissue characterization based on acquiring backscattered echoes from the heated breast. We have proved that the temperature increase inside the breast modifies the intensity, spectrum of the backscattered signals and the probability density function of envelope samples. We discuss the differences in probability density functions in two types of tissue regions, e.g. cysts and the surrounding glandular tissue regions. Independently, Pennes bioheat equation in heterogeneous breast tissue was used to describe the heating process. We applied the finite element method to solve this equation. Results have been compared with the ultrasonic predictions of the temperature distribution. The results confirm the possibility of distinguishing the differences in thermal and acoustical properties of breast cyst and surrounding glandular tissues.
\end{abstract}

Keywords: medical ultrasound; temperature changes in vivo; breast tissue; ultrasonic temperature measurement.

\section{Introduction}

Ultrasound images contain information about the properties of examined tissues; their local acoustic impedance is encoded in grayscale. The diagnostic interpretation of the breast lesions' images is done by a physician on the basis of the BI-RADS (Breast Imaging-Reporting and Data System) classification.

Numerous new methods of Quantitative Ultrasound (QUS) dedicated to the estimation of the structural changes in tissue are being developed (MAmou, Oelze, 2013; Wu et al., 2015). Particularly, analysis of statistical properties of the backscattered radiofrequency (RF) signals have been successfully applied to differentiate healthy tissue from tissue regions changed pathologically (NowICKI et al., 2015). Up to now, the most reliable diagnostic results were obtained for liver and renal tissues lesions, because their normal, healthy structures are nearly homogeneous. The classification of the strongly heterogeneous breast tissue is however still an open issue (BYRA et al., 2016). Recent study revealed that the medium contraction and expansion induced by a temperature change results in relative displacement of the scatterers in heated tissue. On the other hand the temperature rise modifies the absorption and changes also the reflectivity of scatterers due to a reversible conversion of protein microstructures. So, all together, these tissue properties modification could potentially enable the non-invasive mapping of the temperature changes by tracking the variations in backscattered ultrasound signals.

In the last decade, numerous studies have been devoted to finding the relationship between changes in temperature and properties of RF backscattered echoes from tissue samples. Various QUS methods were used for the detection of the temperature distribution within the sonificated volume, e.g. (Doubrovina et al., 2014; Kruglenko, Gambin, 2014; Byra, GamBin, 2015; Gambin, Kruglenko, 2015). 
In general, these methods belong to "ultrasonic thermometry", well documented research field, (see references in the latest review article LEWIS et $a l ., 2015)$.

There are many articles on the use of ultrasonic thermometry in tissue regions in vitro, but to our knowledge there are no published results for human breast tissues in vivo, except our two papers published recently (GAMBIN et al., 2015a; 2015b). Previously, we have studied thermal variation in statistical distributions of the echoes' amplitudes recorded in soft tissue phantoms and samples of soft tissue (a pig liver and chicken breast) in vitro.

The problem of temperature distribution inside the breast tissues was extensively studied in the foundations of thermography imaging. The numerical models applied to theoretical and numerical modelling of the temperature fields inside the breast tissue with different lesions in normal body temperature conditions were proposed in (BALUsu et al., 2014; GonzÁlez, 2007; KWoK, Krzyspiak, 2007; NG, Sudharsan, 2001; Subhadeep, BAlaji, 2010), where the assumptions on the existence of differences in heat production in healthy and diseased tissues were assumed. The main aim of the research reported in the publications mentioned above was to improve the detection of neoplastic lesions in the breast by measuring the temperature distribution on the breast skin surface.

We took a different approach. The skin surface was heated in vivo and backscatter RF ultrasonic echoes from the tissue before and after the heating were recorded.

The information on the type of scattering tissue structures is encoded in the statistics of the echoes amplitudes from defined regions of interest (ROI). It is well known that when a signal is backscattered from a medium with a large number of uniformly distributed scatterers then the random amplitude fluctuations are close to the Rayleigh distribution. Derogation of that distribution, due to more heterogeneous scatterers distribution, e.g. the appearance of cell clusters or/and the scatterers reflectivity variations, results in the amplitude distribution better modeled when using more complex probability distribution, such as K, Nakagami or Gamma distributions.

In this paper two algorithms, the minimum likelihood estimation and method of moments, fitting the amplitude histograms to different probability distribution functions (PDF) for the cyst regions and glandular tissue regions are used. The relationships between PDFs and local temperature are discussed. Additionally, we have compared both, signal intensities and spectrum variations resulting from the temperature rise in cyst and surrounding tissue regions.

In order to explain links between the temperature field predicted by the numerical analysis and spatial variations of changes in backscattered energy (CBE), the spatial distribution of temperature based on finite elements method (FEM) solution of Pennes bioheat equation was calculated.

The paper is organized as follows: Sec. 2 describes the experimental setup and methods applied, Sec. 3 presents the results, and the final conclusions are given in Sec. 4.

\section{Material and methods}

\subsection{Experiment description}

Backscattered ultrasound RF signals and B-mode images have been collected using ULTRASONIX, (SonixTOUCH, Canada) scanner, 7.5 MHz frequency linear array L14-5/38 transducer, focused at the depth $3.5 \mathrm{~cm}$. The breast was scanned before and after being heated by physician and the lesion region to be analyzed was chosen. The heating process was done directly through the skin to which a rubber seal bag with hot water at $\mathrm{c} / \mathrm{a} 50^{\circ} \mathrm{C}$ during 6 minutes was applied.

\subsection{Methods}

The US images (before and after heating) were obtained by an experienced physician with 15-year practice in US breast examinations, see Fig. 1.

The RF signals processing was done using Matlab software (Mathworks Inc., Natick, Massachusets, USA). The scan lines were compensated for the attenuation and the amplitudes of all RF lines were calculated using the Hilbert transform. The backscattered signal amplitudes from the cyst and gland regions for two different thermal conditions were used for statistical analysis of the scattering properties. At first, different PDFs were fitted to the amplitude histograms of the backscattered echoes.

The accuracy of fitting for different PDFs was measured with the mean squared error (MSE). Similarly, the spectrum of mean signals backscattered from the cyst and gland regions in two different thermal conditions was calculated using the FFT (Fast Fourier Transform) in order to differentiate spectral properties related to the thermal changes in cyst and glandular regions. The CBEs from the different tissue regions were calculated and compared with the temperature rise calculated using FEM model.

The numerical model of bioheat transport, descried in details in (GAMBIN et al., 2015a) was used to localize the regions with temperature variations. The FEM solution of $2 \mathrm{D}$ boundary value problem for Pennes equation with perfusion and metabolic heat generation terms was used to simulate the experimental conditions. Independently, we calculated the changes in the $\mathrm{CBE}$ in several regions. We used the $\mathrm{dB}$ measure of CBE which we define below. 

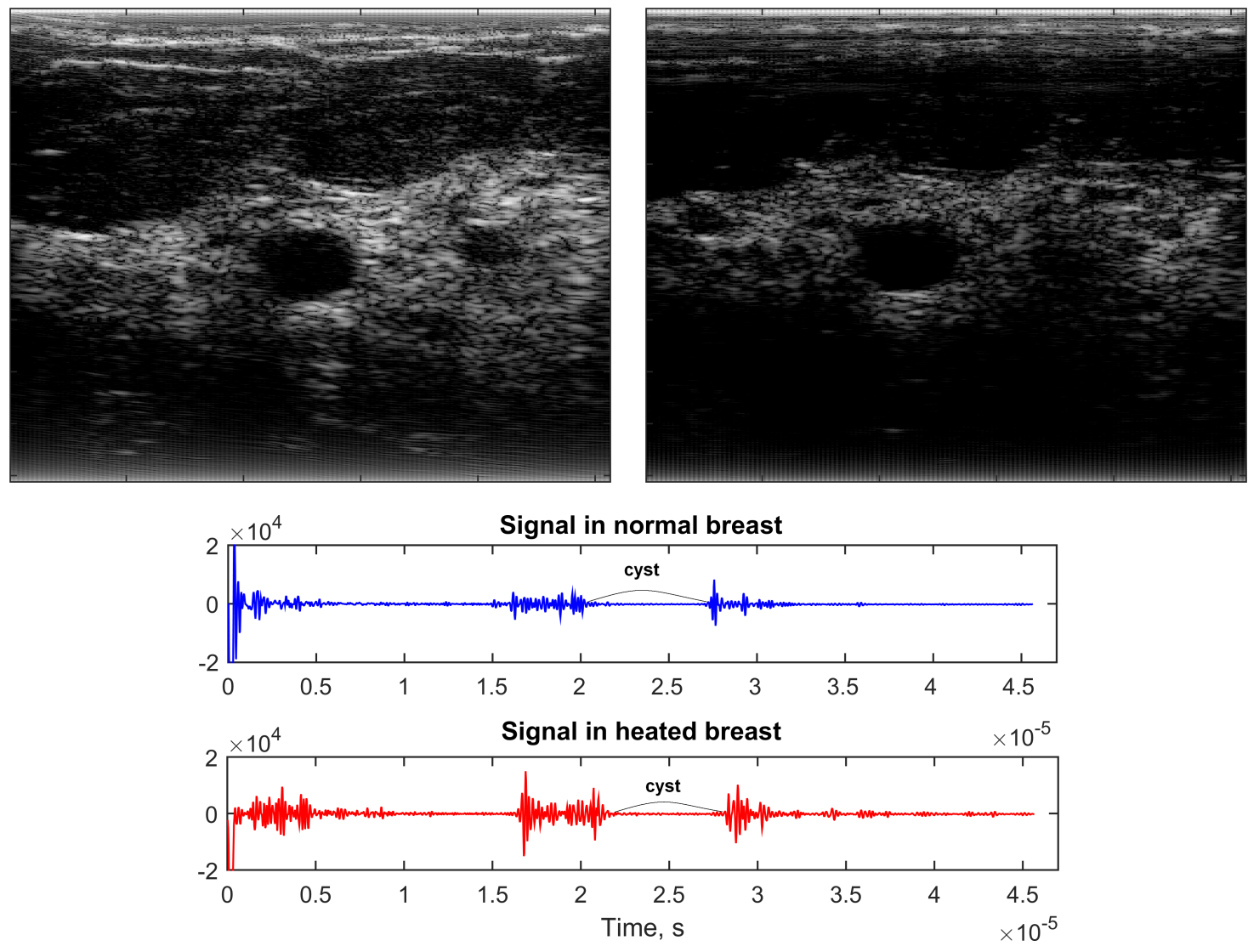

Fig. 1. B-mode images of the breast. Left - normal temperature and right -6 minutes after heating. Below, the examples of raw RF signals from the normal and heated breast.

\section{Results}

\subsection{Temperature distribution}

To determine the temperature field inside the breast in vivo a numerical FEM model was used (GAMBin et al., 2015a) and implemented in the Abaqus 6.12 software environment (DS SIMULIA Corp.). A general bioheat transfer equation in an inhomogeneous thermally anisotropic medium, occupying domain in the real space, may be written as:

$$
\begin{aligned}
\rho(x) C(x) & \frac{\partial T(x, t)}{\partial t}=\partial_{i} K(x) \cdot \partial_{i} T(x, t) \\
& +Q_{p}(x, t)+Q_{m}(x, t)+Q_{\text {ext }}(x, t),
\end{aligned}
$$

where summation index $i=1,2,3$ in 3D real space, $T$, $t, \rho, C, K, Q_{p}, Q_{m}$ and $Q_{\text {ext }}$ denotes temperature, time variable, density, specific heat, thermal conductivity of the medium, blood perfusion term, metabolic heat generation term and external heating. Perfusion term has the form:

$$
Q_{p}(x, t)=\omega_{b} C_{b} \rho_{b}\left(T_{a}-T\right),
$$

where $\omega_{b}, \rho_{b}, C_{b}$ and $T_{a}$ denotes blood perfusion rate, density of blood, specific heat of blood and arterial blood temperature and the coefficients depending on the variable $x$.
The medium under consideration consists of four materials occupying the domain $V=V_{s} \cup V_{f} \cup V_{b} \cup V_{c}$, $V_{s}$ - skin, $V_{f}$ - fat, $V_{b}$ - breast gland and $V_{c}$ - breast cyst, respectively. Then, in Eq. (1) the three coefficients, $\rho(x), C(x)$ and $K(x)$ take the form

$$
\begin{gathered}
\rho(x)= \begin{cases}p_{s} & \text { for } x \in V_{s}, \\
p_{f} & \text { for } x \in V_{f}, \\
p_{b} & \text { for } x \in V_{b}, \\
p_{c} & \text { for } x \in V_{c},\end{cases} \\
C(x)= \begin{cases}C_{s} & \text { for } x \in V_{s}, \\
C_{f} & \text { for } x \in V_{f}, \\
C_{b} & \text { for } x \in V_{b}, \\
C_{c} & \text { for } x \in V_{c},\end{cases} \\
K(x)=K(x)= \begin{cases}K_{s} & \text { for } x \in V, \\
K_{f} & \text { for } x \in V_{s}, \\
K_{b} & \text { for } x \in V_{b}, \\
K_{c} & \text { for } x \in V_{c},\end{cases}
\end{gathered}
$$

where $I$ denotes unit second order tensor. 
Table 1. Material properties of the breast tissue model used in numerical calculations (http://www.itis.ethz.ch/virtual-population/tissue-properties/database/heat-transfer-rate/).

\begin{tabular}{|l|c|c|c|c|c|}
\hline & $\begin{array}{c}\text { Density } \\
{\left[\mathrm{kg} / \mathrm{m}^{3}\right]}\end{array}$ & $\begin{array}{c}\text { Conductivity } \\
{\left[\mathrm{W} /\left(\mathrm{m}^{\circ} \mathrm{C}\right)\right]}\end{array}$ & $\begin{array}{c}\text { Specific heat } \\
{\left[\mathrm{J} /\left(\mathrm{kg}^{\circ} \mathrm{C}\right)\right]}\end{array}$ & $\begin{array}{c}\text { Metabolic heat } \\
\text { generation }\left[\mathrm{W} / \mathrm{m}^{3}\right]\end{array}$ & $\begin{array}{c}\text { Blood perfusion } \\
\text { rate }[\mathrm{ml} / \mathrm{s} / \mathrm{ml}]\end{array}$ \\
\hline Breast gland & 1060 & 0.42 & 3540 & 700 & 150 \\
\hline Breast fat & 911 & 0.21 & 2348 & 450 & 47 \\
\hline Breast cyst & 1000 & 0.6 & 4200 & 0 & 0 \\
\hline Skin & 1109 & 0.37 & 3391 & 368 & 10000 \\
\hline Blood & 1050 & 0.6 & 3800 & - & \\
\hline
\end{tabular}

2D image (see Fig. 2) of tissue scan used in the numerical model included the skin layer (skin) of $2 \mathrm{~mm}$, unevenly distributed fatty tissue of 2-9 $\mathrm{mm}$, gland

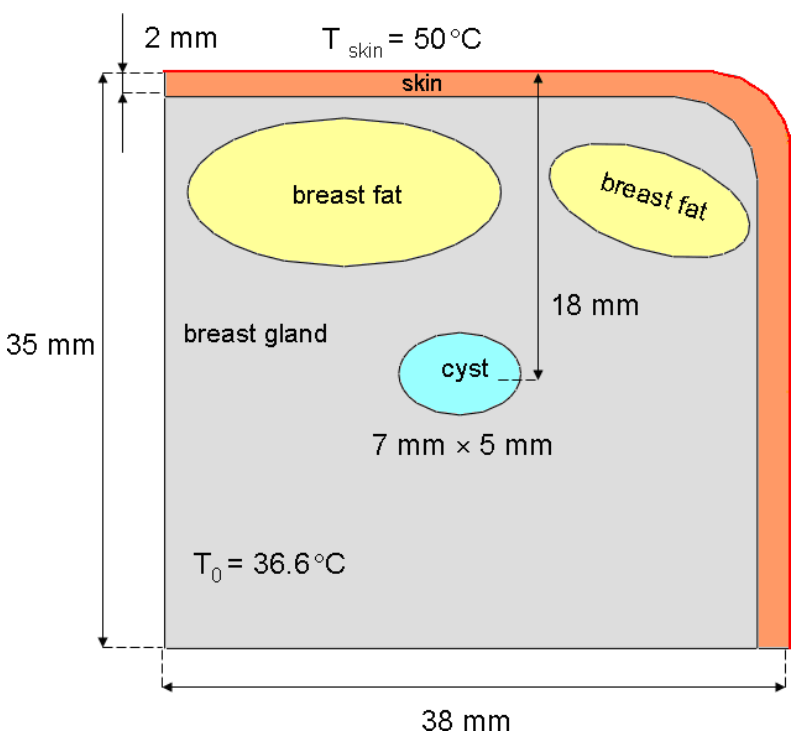

Fig. 2. 2D simplified geometrical model used in FEM calculations. breast tissue, and the cyst filled with fluid of about $5 \mathrm{~mm} \times 7 \mathrm{~mm}$. The cyst was located at a distance of $18 \mathrm{~mm}$ from the skin surface. The above given the general bioheat transfer equation, describing the temperature field generated by a surface heat source on the skin surface and with the assumed constant temperature on the rest of the boundary, without the external volume heat sources was numerically solved.

The initial temperature in the whole area was assumed to be equal $37^{\circ} \mathrm{C}$. After 6 min heating the temperature on the skin boundary increased up to $42^{\circ} \mathrm{C}$. Material parameters for numerical model are given in Table 1.

The temperature distribution after heating is shown in Fig. 3. Let us notice, that the temperature rise inside the breast is heterogeneously distributed. Thermal properties of the skin, assumed in our theoretical model, sufficiently protect the internal part of breast to preserve safety temperature variations.

\subsection{Spectral properties temperature variations}

The mean spectra of the RF data lines from two different breast regions $3 \mathrm{~A}$ and $3 \mathrm{~B}$, marked in Fig. 3, and

a)

b)
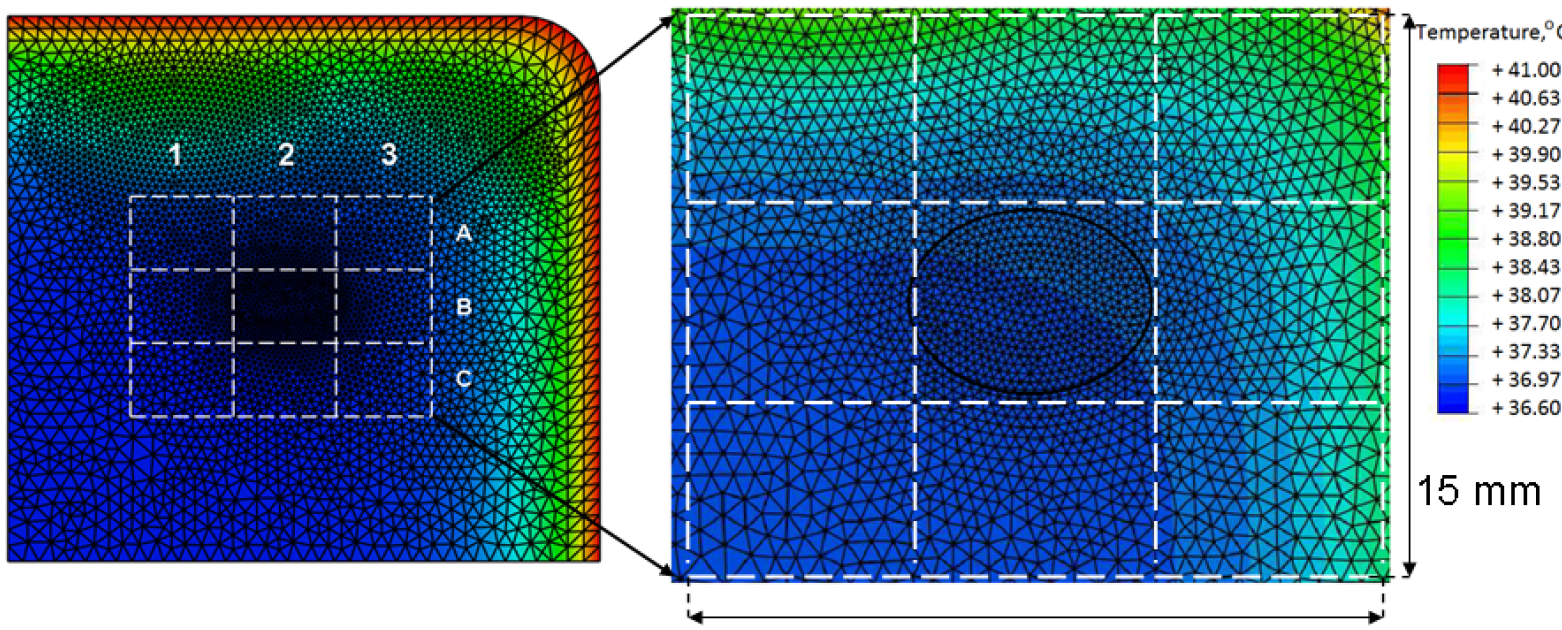

$21 \mathrm{~mm}$

Fig. 3. a) temperature distribution within breast tissue calculated using FEM, b) enlarged region with better visibility of the temperature distribution in the tissue surrounding the cyst. The mean temperature rise in all nine marked regions was calculated. 

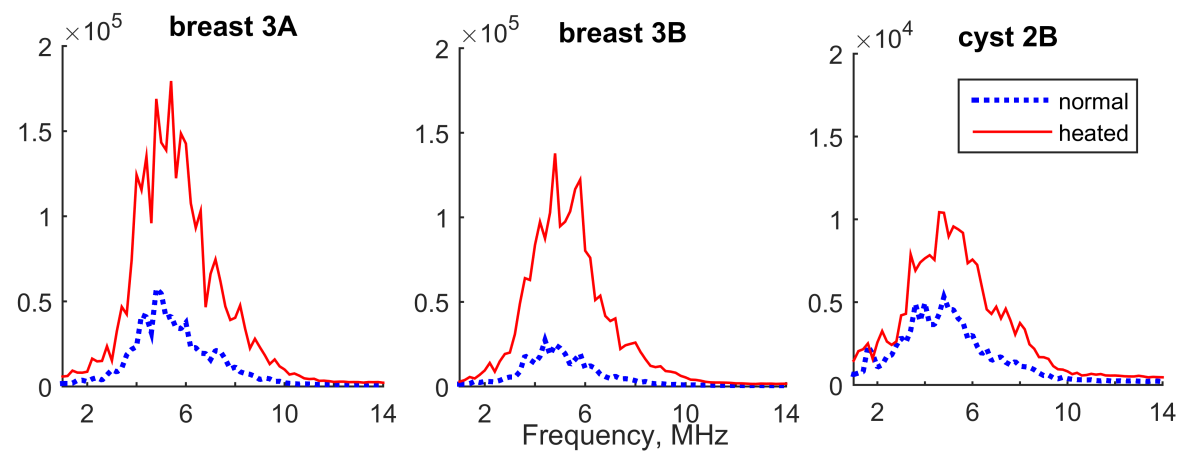

Fig. 4. Mean frequency spectrum in the two tissue regions, left, and right before and after heating.

from the cyst region were calculated. The dimension of regions was $5 \times 4 \mathrm{~mm}$, which corresponds to $35 \mathrm{im}$ age lines with 200 samples each. The spectrum was calculated for every line and then averaged. Specific behaviour of backscattered signal spectral properties due to the temperature variations is shown in Fig. 4. The amplitude values are 10 times greater in the breast regions than in the cyst. In the breast US scan (Fig. 1) the cyst region is darker than the surrounding tissue.

\subsection{Statistical properties in function of temperature variations}

Statistical properties of the RF envelope from different ROI's were calculated by fitting the histograms to different PDF's, namely Nakagami, K-distribution, Gamma and Rayleigh. The goodness of fit was determined using MSE. The breast tissue regions used in calculation of histograms are denoted as A2, A3, B3, $\mathrm{C} 2$ and $\mathrm{C} 3$ in Fig. 3. For improved statistical results, the cyst was also divided into the five different regions, extracted randomly from the cyst, with equal areas, overlapping in c/a $50 \%$.

The Nakagami PDF revealed the smallest MSE for all breast tissue regions. Hence, we used the Nakagami shape parameter $m$ as the best factor characterizing the scattering properties of breast tissue regions. The heated regions revealed significantly lower values of the Nakagami shape parameter. Shape parameters of the K-distribution and Gamma distribution can also be considered as a qualitative measure of the scattering character and they were also lower for heated tissues. The Rayleigh parameter was at least 3 times larger in the heated regions, due to the increased intensity of the diffusive scattering. Inside the cyst regions, at the normal temperature level, the histograms are best fitted to the Nakagami distribution. In the heated cyst all the distributions fitted to the histograms were very similar to each other in the MSE sense. Hence, any of these distribution can be used to characterize cyst backscattering properties. It should be noticed that shape parameters for all examined distributions of the backscattered echoes from the cyst regions were significantly larger after heated. The graphical illustration of the above discussion is presented in Figs. 5
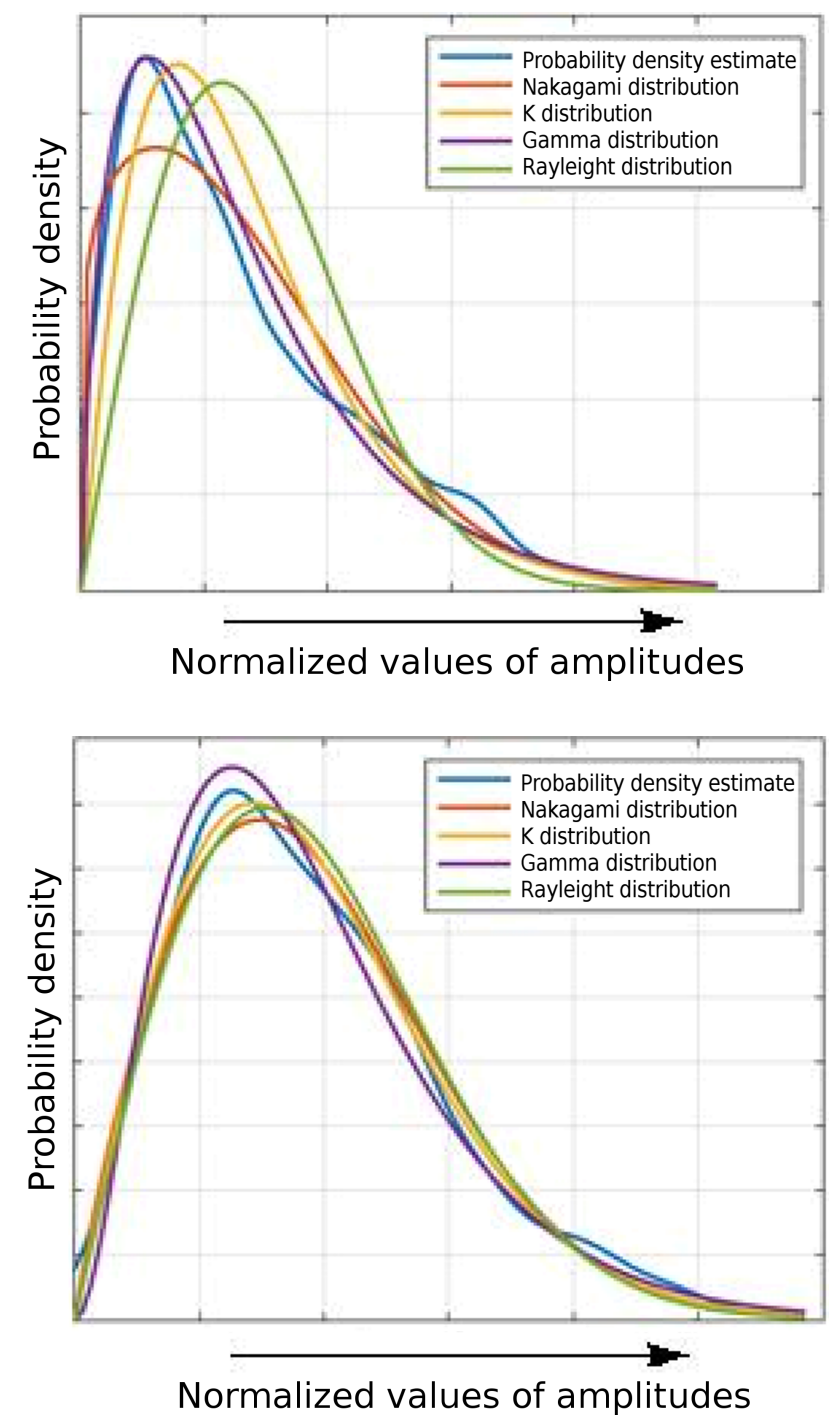

Fig. 5. PDF estimates of signals amplitudes collected from the glandular tissue region and four PDFs fitted to histograms, top - normal temperature region, bottom - heated region. 
and 6. For clarity, envelope samples in each data set were scaled to $[0,1]$. The impact of the temperature rise on the smoothed histograms and their estimates by the different probability density functions, calculated from the one glandular tissue region, A2, and the cyst region, B2, are shown in Fig. 5 and Fig. 6, respectively. Both, for the tissue region and for the cyst, most of the histogram estimations were "far" from the histogram and they started to be get closer after heating.
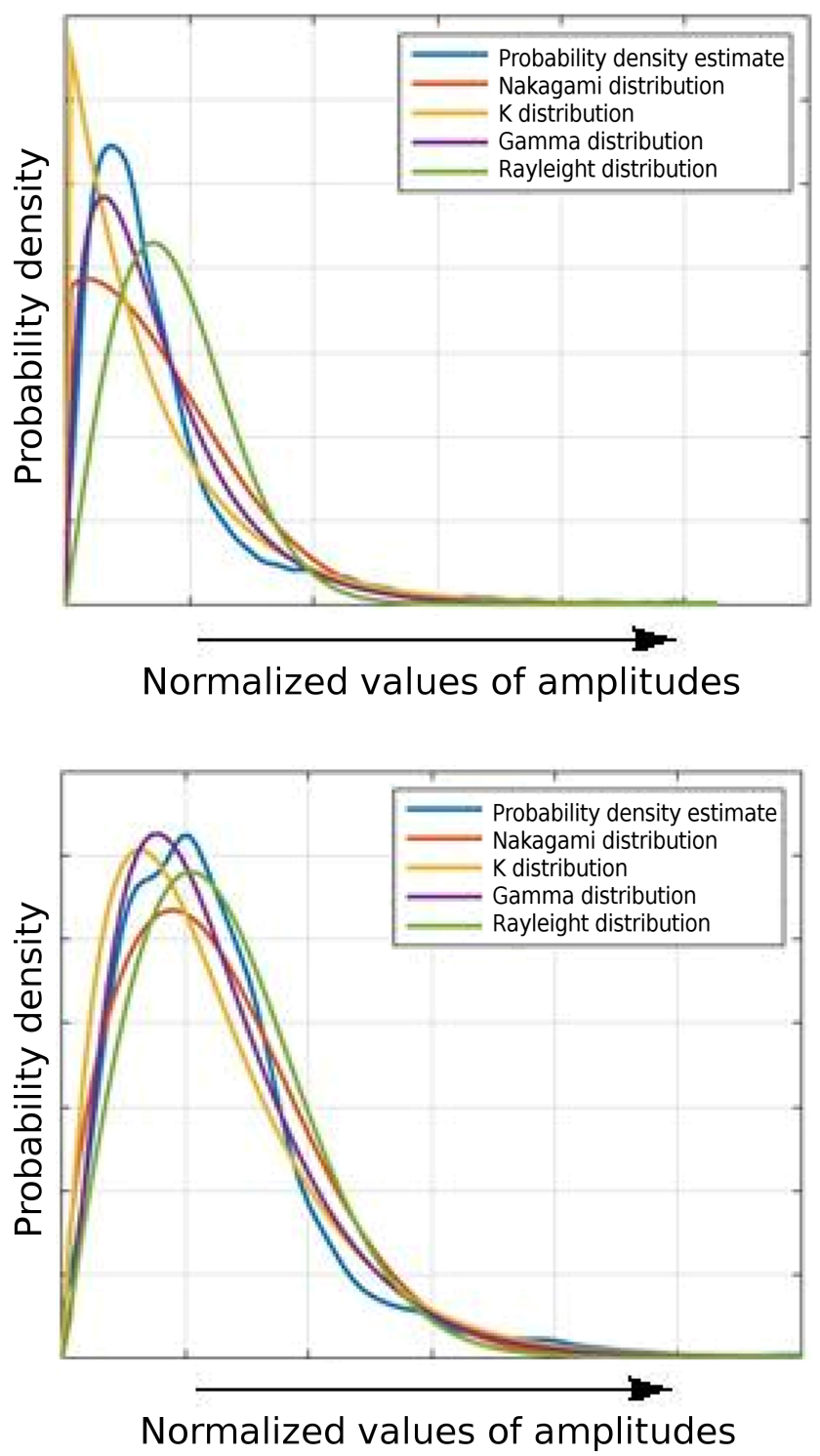

Fig. 6. PDF estimates of signals amplitudes collected from the cyst region and four PDFs fitted to histograms, top normal temperature region, bottom - heated region.

For better illustration of statistics variations in cyst and surrounding tissue due to the temperature rise, $2 \mathrm{D}$ graphs of the Nakagami shape parameter versus SNR (signal to noise ratio) are plotted in Fig. 7.

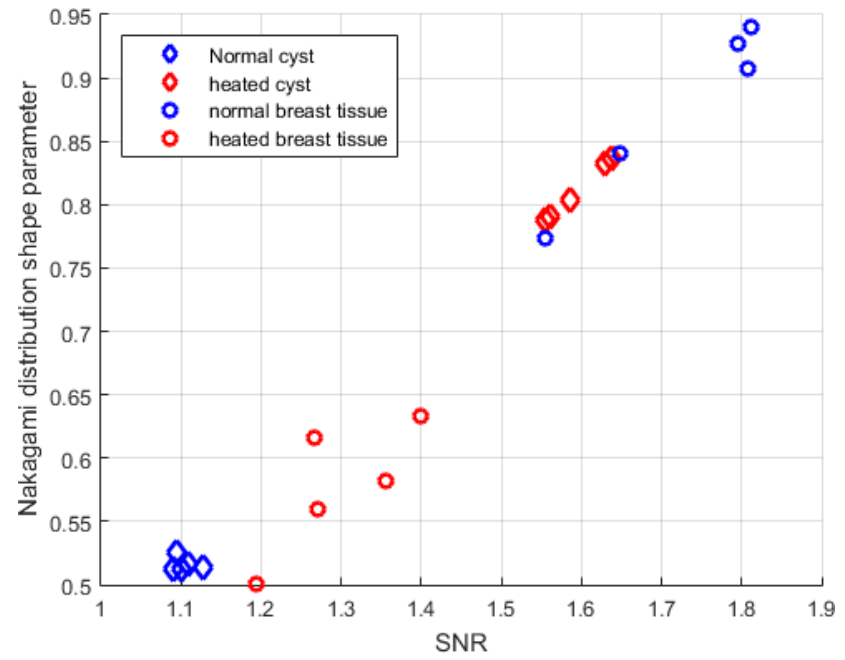

Fig. 7. Values of the Nakagami shape parameter versus SNR in five breast tissue regions and in five cyst regions before (black) and after heating (gray).

\subsection{Thermal changes in backscattered energy}

Previously, we have reported the changes in backscattered energy CBE (for the same experimental data) in the cyst and surrounding regions calculated as the ratio of squared amplitudes before and after heating (GAMBIN et al., 2015b). Here, we calculate the CBE in dB scale for the initial, "normal", temperature $T_{0}$ and, after heating, at temperature $T$. The backscattered echoes' amplitudes were squared (value proportional to echoes intensity) and CBE for each pixel $(i, j)$, where $i$ is a sample number and $j$ is an image line, has been calculated as follows (Tsui et al., 2012):

$$
C B E(i, j)=10 \log _{10} \frac{\operatorname{env}_{T}^{2}}{\operatorname{env}_{T_{0}}^{2}}(i, j),
$$

where $\operatorname{env}_{T_{0}}$ and $\operatorname{env}_{T}$ denote envelopes of signals at the initial temperature and actual temperature, respectively. The envelopes are calculated from the firstly



Fig. 8. Maps of CBEs with spatial resolution, $2 \times 2 \mathrm{~mm}$. 
obtained analytical signals with the help of Hilbert transform of raw signals.

The CBE, in $[\mathrm{dB}]$ in different regions was calculated by averaging over all pixels $(i, j)$ belonging to the region. The maps of CBEs with $2 \mathrm{~mm}^{2}$ spatial resolution, is presented in Fig. 8.

In order to compare the CBE maps and numerical temperature distribution obtained using FEM model, we have calculated the average temperature values in all 9 regions (Fig. 2), and the results are plotted in Fig. 9.
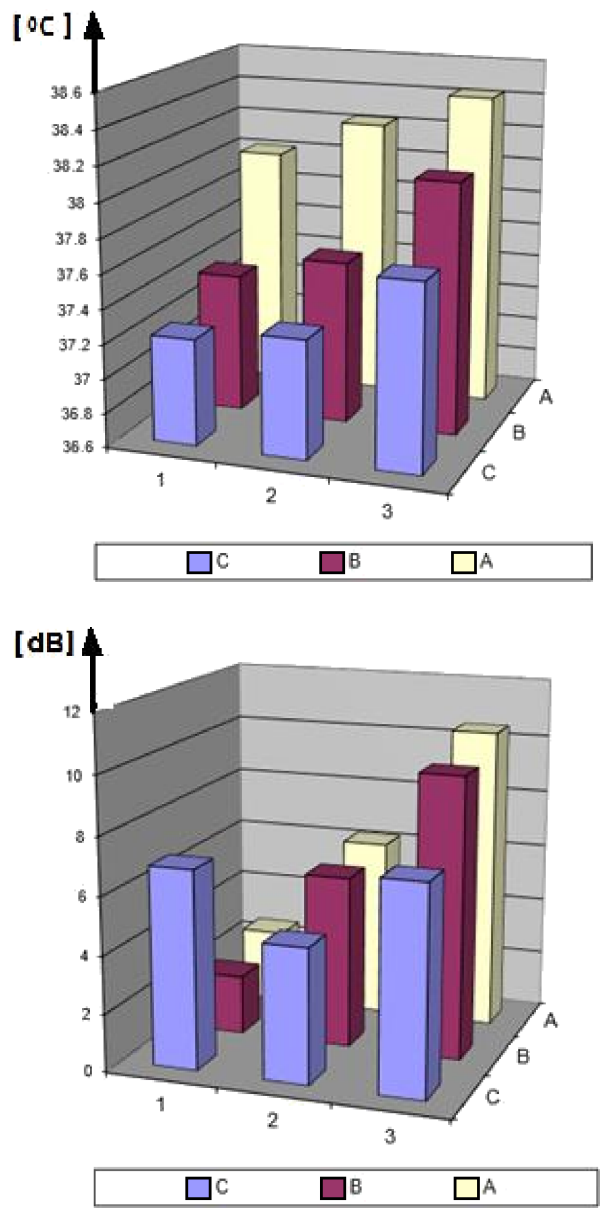

Fig. 9. The mean temperature distribution in 9 regions from Fig. 2 calculated using FEM (top), and the mean values of $\mathrm{CBE}$ in the same regions (bottom).

\section{Conclusions}

The quality of B-mode images was significantly improved after heating of breast. The fine details of the cyst structure were clearly visible while the same region before heating was rather hypo-echogenic. The penetration depth was increased by over $1 \mathrm{~cm}$ comparing to the pre-heating scanning (Fig.1). It means that the RF echo amplitude after heating is larger. In the less heated regions (left of the cyst) there are no visible differences in the US image after heating, but the differences occur in calculated from the row data CBE (Fig. 9).

It should be stressed that the values of statistical parameters are very sensitive to the variations of temperature level, more in the normal tissue regions as compared to the cyst region. Hence, the effect is "opposite" in a sense, that the Nakagami parameters decrease for the heated breast tissue regions whereas they increase within the cyst regions. The full explanation of this effect is still an open issue. Only a partial interpretation can be done. The speed of sound in the cyst, resembling the suspension of scatterers in fluid medium is larger after heating, hence the resolution cell contains more scatterers. We can speculate that the PDFs for the heated cysts are better modeled by the Rayleigh distribution, because the Nakagami shape parameter is close to 1 and SNR is in the range 1.551.65 (Fig. 6). However, there is no such simple explanation in the case of the strongly inhomogeneous breast tissue region, which contains different structures and blood vessels. Our knowledge on the impact of the temperature rise on the microstructural properties inside the living tissue is by far incomplete. But the lower values of Nakagami parameter in heated regions suggest that the scattering patterns are more heterogeneous, than in the tissue in the body temperature. It can be naively explained through the signal power enhancement that causes larger fluctuations in the reflectivity of scatterers.

After heating, the spectrum amplitude increases much more in the surrounding tissue, than in the cyst lesion comparing to the normal temperature level. In glandular tissue regions, the rise of a relative spectrum amplitude level is much greater than in the cyst regions. The changes in the spectrum shape - especially reduction of the bandwidth - are stronger after heating in both breast regions than in the cyst region. The most heated region A3 has higher spectral energy than region $\mathrm{B} 3$. This is also confirmed by FEM model and CBE maps (Fig. 9). We shall also underline nearly perfect agreement between numerical FEM calculations and CBE maps (Fig. 8 and Fig. 9). Only within the one single region $\mathrm{C} 1$, the predictions are in contradiction. It can be caused by the fact that the US breast images recorded before and after heating were obtained not exactly from the same volume of tissues.

Summing up, we have proved that the statistical properties of RF echoes data, the changes in mean echoes spectrum and changes in the backscattered energy, CBE, carry the information allowing to differentiate thermal properties of the breast cyst and surrounding tissue.

\section{Acknowledgment}

This work was partially supported by National Science Centre (2011/03/B/ST7/03347). 


\section{References}

1. Balusu K., Suganthi S.S., Ramakrishnan S. (2014), Modelling Bio-heat transfer in Breast Cysts using Finite Element analysis, International Conference on Informatics, Electronics and Vision, ICIEV, 1-4, Dhaka, Bangladesh.

2. Byra M., Gambin B. (2015), Temperature detection based on nonparametric statistics of ultrasound echoes, Hydroacoustics, 18, 17-23.

3. Byra M., Nowicki A., PiotrzkowskAWróblewska H., Dobruch-Sobczak K. (2016), Classification of breast lesions using segmented quantitative ultrasound maps of homodyned $K$ distribution parameters, Med. Phys. 43, 5561-5569, DOI: 10.1118/1.4962928.

4. Doubrovina O., Gambin B., Kruglenko E. (2014), Temperature level and properties of wavelet approximation of backscattered ultrasound, Hydroacoustics, 17, $37-46$.

5. Gambin B., Kruglenko E., Wójcik J. (2015a), Relationship between thermal and ultrasound fields in breast tissue in vivo, Hydroacoustics, 18, 53-58.

6. Gambin B., Kruglenko E., Byra M., Nowicki A., Piotrzkowska-Wroblewska H., DobruchSOBCZAK K. (2015b), Changes in ultrasound echoes of a breast tissue in vivo after exposure to heat-a case study, Proceedings of 3rd Congress of Mechanics, 1, pp. 217-218, Gdańsk, Poland.

7. Gambin B., Kruglenko E. (2015), Temperature Measurement by Statistical Parameters of Ultrasound Signal Backscattered from Tissue Samples, Acta Physica Polonica, 128, A-72-A-78.

8. GonzÁlez F.J. (2007), Thermal simulation of breast tumors, Revista Mexicana de Física, 53, 4, 323-326.

9. Kruglenko E., Gambin B. (2014), RF signal amplitude statistics during temperature changes in tissue phantoms, Hydroacoustics, 17, 115-122.
10. Kwok J., Krzyspiak J. (2007), Thermal Imaging and Analysis for Breast Tumor Detection, BEE 453: Computer-Aided Engineering: Applications to Biomedical Processes.

11. Lewis M.A., Staruch R.M., Chopra R. (2015), Thermometry and Ablation Monitoring with Ultrasound, Int. J. Hyperthermia, 31, 2, 163-181.

12. Mamou J., Oelze M.L. [Eds.], (2013), Quantitative Ultrasound in Soft Tissues, Dortrecht-HeilderbergNew York-London, Springer.

13. NG E.Y.K., Sudharsan N.M. (2001), An improved three-dimensional direct numerical modelling and thermal analysis of a female breast with tumour, Proceedings of the Institution of Mechanical Engineers, 215, $25-37$.

14. Nowicki A., Piotrzkowska-Wroblewska H., Litniewski J., Byra M., Gambin B., Kruglenko E., Dobruch-SobczAK K. (2015), Differentiation of normal tissue and tissue lesions using statistical properties of backscattered ultrasound in breast, in Ultrasonics Symposium (IUS), 2015 IEEE International, DOI: 10.1109/ULTSYM.2015.0417.

15. Subhadeep M. Balaji C. (2010), A Neural Network Based Estimation of Tumor Parameters from a Breast Thermogram, International Journal of Heat and Mass Transfer, 53, 4714-4727.

16. Tsui P.H., Shu Y.C., Chen W.S., Liu H.L., Hsiao I.T., Chien Y.T. (2012), Ultrasound temperature estimation based on probability variation of backscatter data, Med. Phys., 39, 2369-2385, http://dx.doi.org/10.1118/1.3700235.

17. Wu W.-J., Shih-Wei Lin, Woo Kyung Moon (2015), An Artificial Immune System-Based Support Vector Machine Approach for Classifying Ultrasound Breast Tumor Images, J. Digit Imaging, 28, 5, 576585, DOI: $10.1007 / \mathrm{s} 10278-014-9757-1$. 